\title{
On-demand acoustic methods for time-resolved structural biology
}

Allen Milster Orville $^{1}$, Franklin D. Fuller ${ }^{2}$, Sheraz Gul ${ }^{2}$, Jan Kern ${ }^{2}$, Pierre Aller ${ }^{1}$, Babak Andi ${ }^{3}$, Aaron Brewster ${ }^{2}$, Nicholas Sauter ${ }^{2}$, Vittal Yachandra², Junko Yano ${ }^{2}$

${ }^{1}$ Life Sciences, Diamond Light Source, Didcot, United Kingdom, ${ }^{2}$ Molecular Biophysics and Integrated Bioimaging Division, Lawrence Berkeley National Laboratory, Berkeley, United States, ${ }^{3}$ NSLS-II, Brookhaven National Lab, Upton, United States E-mail: allen.orville@diamond.ac.uk

Acoustic droplet ejection (ADE) is a general, touchless, on-demand method that uses focused sound waves to eject picoliter to nanoliter volume droplets from the surface of one liquid to another location. The frequency of the wave front from a spot-focused piezoelectric transducer influences the ejected droplet size, whereas the amplitude of the sound waves translates into droplet velocity. ADE was originally developed for the biomedical industry, and we have adapted it to create new strategies for serial femtosecond crystallographic (SFX) approaches at XFEL facilities and complementary strategies at synchrotron sources. SFX requires large numbers of diffraction patterns from randomly orientated microcrystals, each yielding one still image before it is destroyed by the X-ray pulse. Consequently, samples of microcrystal slurries must be replenished at a sufficient pace to keep up with the XFEL pulse frequency. To help meet the sample consumption challenge, we and others have been developing on-demand sample delivery methods. By delivering discrete, well-separated drops that are timed to each $\mathrm{X}$-ray pulse, ADE-based methods decrease sample consumption and increase data collection efficiency.

In our first applications at the Linac Coherent Light Source (LCLS), we collected SFX datasets from droplets launched in synchrony to intersect each XFEL pulse [1]. We have also coupled ADE methods with a conveyor belt drive to enable pumpprobe, time-resolved SFX (tr-SFX) at the LCLS [2]. Our system is optimized for crystallography and X-ray emission spectroscopy (XES) measurements of photochemical reactions over a wide-range of time scales and illumination schemes [3]. Indeed, the combination of tr-SFX + XES, wherein both types of data are obtained from the same sample and X-ray pulse, provides important complementary information that impacts mechanistic insights. We are also exploiting the region on the belt between the ADE transducer and the X-ray interaction point to introduce additional experimental perturbations that initiate a range of enzyme reactions. I will discuss the potential impact to tr-SFX studies of many macromolecules operating under physiological temperature and pressure.

A.M.O. acknowledges support from a Strategic Award from the Wellcome Trust and the Biotechnology and Biological Sciences Research Council (grant 102593).

[1] Roessler, C. G. et al (2016) Structure 24, 631-640.

[2] Fuller, F. D. and Gul S. et al (2017) Nature Methods (in press).

[3] Young, I. D., Ibrahim, M., and Chatterjee, R. et al (2016) Nature 540, 453-457.

Keywords: on-demand serial femtosecond crystallography, acoustic injector, pump-probe tr-SFX 